\title{
When Me Is Mine: An Embodied Origin of Psychological Ownership?
}

\author{
Aurora De Bortoli Vizioli (aurora.debortolivizioli@gmail.com) \\ Institute of Cognitive Sciences and Technologies, CNR \\ Via San Martino della Battaglia 44, 00185, Rome, Italy \\ Anna M. Borghi (anna.borghi@uniroma1.it) \\ Department of Dynamic and Clinical Psychology, Sapienza University of Rome \\ Via degli Apuli 1, 00185, Rome, Italy \\ Institute of Cognitive Sciences and Technologies, CNR \\ Via San Martino della Battaglia 44, 00185, Rome, Italy \\ Luca Tummolini (luca.tummolini@istc.cnr.it) \\ Institute of Cognitive Sciences and Technologies, CNR \\ Via San Martino della Battaglia 44, 00185, Rome, Italy
}

\begin{abstract}
Neurological evidence has shown that brain damages can selectively impair the ability to discriminate between objects belonging to others and those that we feel are our own. Despite the ubiquity and relevance of this sense of object ownership for our life, the underlying cognitive mechanisms are still poorly understood. Here we ask whether psychological ownership of an object can be based on its incorporation in one's body image. To explore this possibility with healthy participants, we employed a modified version of the rubber hand illusion in which both the participant and the rubber hand wore a ring. We used the self-prioritization effect in a perceptual matching task as an indirect measure of the sense of (dis)ownership over objects. Results indicate that undermining the bodily self has cascade effects on the representation of owned objects, at least for those associated with the body for a long time.
\end{abstract}

Keywords: psychological ownership; body-ownership; rubberhand illusion; bodily self; extended self.

\section{Introduction}

During our daily interactions, we often rely on our ability to identify, and differentially treat, what belongs to us from what belongs to others. Importantly, this sense of ownership of possessions is malleable. Sometimes, a tacit sense that something is ours instantaneously develops (think of the rapid way in which we acknowledge 'our' cutlery at the restaurant; Scorolli et al, 2018). Other times, it is our prolonged use of an object, like the unconstrained access to a friend's bicycle, that generates a feeling that a piece of someone else's property seems to belong to us. The societal relevance of this phenomenon has been long recognized by the legal doctrine of adverse possession that acknowledges that legal rights of ownership can be acquired by occupation or possession of someone else's property (Merril, 1984). Given its effects on the wellbeing of individuals, and its potential to foster caring and assumption of responsibility for resources that are not actually owned by any individual in particular, this phenomenon is also relevant for psychologists (Peck \& Shu, 2018). To measure it, the construct of "psychological ownership" - defined as the state in which individuals feel as if the target of ownership is 'theirs' (Pierce et al, 2003) - has been postulated. The interdisciplinary literature adopting this construct has identified at least three antecedents for this feeling: (1) an investment of the self in the target; (2) its intimate knowledge; (3) the ability to control it (Pierce et al, 2003). Besides the identification of these boundary conditions, however, the cognitive mechanisms that support psychological ownership have not been so far explored.

The first of these antecedents, on which this work is based, was inspired by a venerable and influential view (James, 1890; Belk, 1988), according to which our conceptual representation of the self can be extended to include also external stimuli that are personally relevant, like, for instance, our own possessions. In this perspective, a sense of ownership can derive by an implicit (and potentially fast) association with the self-concept (Ye \& Gawronski 2016), i.e. (what is) mine is (also part of) me. Consistently with this mine is me view, it has been shown that the representation of self-owned objects enjoys the same processing priority of other stimuli associated with the self and recruits brain regions involved in self-referential processing (Turk et al., 2011b; Kim \& Johnson, 2014). Such fast association with the self-concept can also potentially explain other cognitive effects of ownership like our tendency to assess our own objects as more attractive (mere ownership effect, Beggan, 1992) and more valuable (endowment effect; Morewedge \& Giblin, 2015), their perceptual and attentional salience (Sui et al 2012; Turk et al., 2011a) and our mnemonic advantage even when ownership is only arbitrarily assigned (Cunningham et al., 2008). Finally, neurological evidence has reported cases of people who describe experiences of loss of ownership feelings toward home objects or pets after brain damage (Nascimento Alves et al, 2016) or after psychotic episodes (Abed and Fewtrell 1990); these are precisely the kind of 
personal belongings typically included in one's extended self.

More recent approaches to the self, however, have demonstrated that its mental representation comprises also perceptual and motor information constituting the so-called minimal or bodily self (Blanke et al, 2015), and not only conceptual information (the semantic and episodic knowledge relevant for the narrative and autobiographical self). Importantly, both the conceptual and the bodily self are malleable and interlinked (Maister et al, 2015).

Thus, besides projecting our (conceptual) self onto external objects (mine is me), we hypothesize that psychological ownership can also result from a bottom-up process in which what becomes part of the (bodily) self is perceived as one's own. In other words, the feeling of ownership over objects might also be grounded on their incorporation into our body image: i.e. (what is perceived as part of) me is mine.

Preliminary indirect support for this view derives from experimental evidence with the "rubber hand illusion" paradigm (RHI, Botvinick and Cohen, 1998) showing that even non-corporeal objects that are not perceptually similar to body parts can become incorporated (Armer \& Ramachandran 2003; Ma \& Hommel, 2015). Weser et al. (2017), for instance, used a novel RHI paradigm in which both the participant's and the rubber hand were equipped with tools (chopsticks). Results showed that applying the visuo-tactile synchronous stimulation on the tip of tools (instead of applying it on the rubber and real hand) was sufficient to induce the characteristic illusion. Whether the illusion also induced a sense of psychological ownership over the incorporated objects however is not known.

More direct evidence that incorporation of an object influences its psychological ownership has been collected by Aglioti and colleagues (Aglioti et al, 1996). The authors report the case of a somatoparaphrenic patient who not only denied ownership of her left hand but also displayed selective disownership of objects typically associated with it (e.g. a wedding ring, a garnet ring, a watch etc.). This delusional disownership only manifested when the ring, for instance, was worn on the affected hand. When the ring was moved from the left to the right hand or placed in the extrapersonal space, the patient was able to correctly recognize it as her own and to access the associated autobiographical information. On the contrary, ownership of other objects belonging to the patient but not commonly associated with the left hand (comb, earrings, small gold pin) were correctly recognized, irrespective of where they were viewed. While this study has been seminal to initiate a systematic exploration of the plasticity of the body schema, it has been less appreciated that it also gathered preliminary evidence that a sense of disownership of body parts can affect the psychological ownership of objects associated with it. Still, whether the inverse is also true has not been so far established.

\section{The Current Study}

In this study, we hypothesize that psychological ownership of an object, for example a piece of jewelry, is partially based on the incorporation of the object in our body image (embodiment) and that changing the bodily selfrepresentation may have a cascade effect on the ownership feelings toward that object.

Previous research has shown that manipulating body appearance can affect other mental processes, such as implicit attitudes, perceptual processing, decision-making and affective judgments (see Maister et al., 2015 for a review). Inspired by these paradigms, here we investigate whether a body-ownership illusion could interfere with the processing of an owned/not-owned object. To manipulate the sense of object- and body-ownership, we employed a modified version of the rubber hand illusion, in which the participant and the rubber hand both wore a ring. Participants wore their own ring while the rubber hand wore an unfamiliar ring. In the classical RHI, participants see a rubber hand being stroke either in synchrony or asynchrony with their own out of view hand. The synchronous condition typically induces the incorporation of the rubber hand into people's body representation, with participants reporting a sense of ownership for the fake hand (see Ehrsson 2012 for a review). Moreover, it has been suggested that experiencing the RHI not only produces embodiment of a fake body part, but also an illusion of disembodiment of the real hand as documented by self-reports (Lane et al., 2017) and physiological measures (Della Gatta et al., 2016). By using a "rubber" ring, we aimed to create an illusion of ownership over the ring worn by the rubber hand and, conversely, an illusion of dis-ownership over the self-owned ring. We hypothesize that, if the synchronous stimulation produces both a sense of ownership toward the fake hand and a sense of dis-ownership toward the real hand, this could also affect the way participants experience the ownership of the two rings (owned/not-owned). Specifically, participants might experience a sense of ownership toward the rubber ring, a sense of dis-ownership toward their own ring, or both.

To measure psychological ownership and its modulation, we used a perceptual matching task, adapted from Sui et al. (2012), in which participants had to determine whether the picture of an object (either self-owned or other-owned) was associated to a self- or other-related label (i.e., ME/OTHER). It is well established that people process differently self-related information compared to otherrelated stimuli, with enhanced processing priority for the self. The self-prioritization effect has been observed not only for highly familiar self-related stimuli such as our face or our name, but also for newly formed and arbitrary associations between the self and a specific class of stimuli (for a review see Humphreys \& Sui, 2015). Results showed a self-prioritization effect in which participants are faster and more accurate when judging correct shape-label pairs associated with self than correct pairs associated with others. 
Although we do not expect a disruption of selfprioritization effect due to the body-(dis)ownership illusion during the synchronous RHI, we hypothesize that the illusion will modulate response times for both self- and other-related matched trials. Specifically, if the illusion of ownership of the fake hand extends to the ring associated with it (not-owned ring), we should observe slower response times in synchronous than in asynchronous group when perceiving objects owned by someone else (Ownership Hypothesis). Similarly, if the illusion of dis-ownership of the biological hand extends also to the ring associated with it (owned ring), we should observe slower response times in synchronous than in asynchronous group when perceiving self-owned objects (Dis-ownership Hypothesis).

\section{Method}

Before the start of the experiment, demographic information and photos of each participant's owned objects (i.e., ring owned by participant, back and palm of left hand with and without ring) were collected and used as stimuli in the perceptual matching task.

\section{Participants}

Forty-two female students (mean age $=23.4$ years, $\mathrm{SD}=$ 3.9 , all but one right-handed, with normal or corrected-tonormal vision) participated after providing written informed consent. From the initial sample, one participant was excluded due to technical errors. The eligibility criterion requiring individuals to wear a ring on their left hand was not revealed to the participants. The study was approved by the ethic local committee (CNR-ISTC N. 000135 $19 / 01 / 2018$ ) and in accordance with the Helsinki Declaration.

\section{Apparatus and Materials}

Perceptual Matching Task The experiment was run on a PC using E-prime software (version 2.0). The stimuli were displayed on a 15.6-inch monitor $(1366 \times 768$ at $60 \mathrm{~Hz})$. Stimuli were presented on a white background. Participants were asked to assess the match-mismatch of picture-label combinations. Each trial consisted of a blank screen (100 $\mathrm{ms}$ ) followed by a label displayed on the top of the screen. After $500 \mathrm{~ms}$, the picture of an object appeared at the center. Both the label and the picture remained on the screen until participants made a response or after $1500 \mathrm{~ms}$ had elapsed.

The task included two labels (i.e., the Italian label " $m e$ " [me] as a self-related stimulus and the Italian label "altro" [other] as a non-self-related stimulus) and 14 objects belonging either to the self- or to the other-ownership category. The seven objects belonging to the self-ownership category were three pictures of the participant's ring hereafter self-owned ring - made from three different angles, and four pictures of the participant's back and palm of the hand, both with or without the self-owned ring. The seven objects belonging to the other-ownership category were three pictures of the rubber hand wearing a ring - hereafter rubber ring - made from three different angles and four pictures of the back and palm of the rubber hand, both with and without the rubber ring. See Figure 1 for an example.

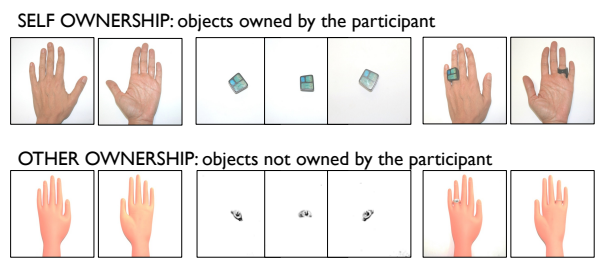

Figure 1: Example of the stimuli included in the selfownership (top) with pictures of the participant's hand and ring and in the other-ownership (bottom) category with pictures of the rubber hand and ring.

Participants were instructed to respond by pressing a right-hand key (M) in case of matched pairs (i.e., "me" and objects included in the self-ownership category or "other" and objects included in the other-ownership category), and a left-hand key (V) in case of non-matched pairs (i.e., "me" and objects included in the other-ownership category or "other" and objects included in the self-ownership category). For left-handed participants the keys were inverted (left-hand key for matched associations and righthand key for unmatched associations).

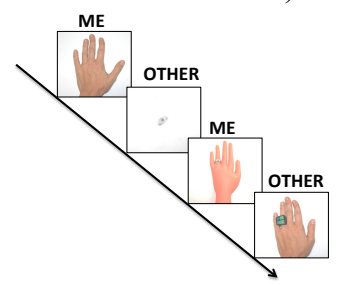

Figure 2: A visual representation of the perceptual matching task. In the first two trials, the pictures match with the labels while in the third and fourth trial they mismatch.

The task was composed by three identical blocks $(1,2,3)$ with block 1 preceded by a 28 trials practice phase. In each block, each of the two labels was associated three times with each of the 14 objects, for a total of 84 trials. The order of presentation of trials was randomized across participants.

Rubber Hand Illusion Participants sat at a table in front of the experimenter, with their left hand wearing the ring placed inside a box, while the rubber hand with the rubber ring was placed on the upper cover of a box. The box measured $19 \mathrm{~cm}$ in width, $10 \mathrm{~cm}$ in height, and $29 \mathrm{~cm}$ in depth. Both the front and the back of the box were removed, allowing participants to place their hand inside the box and the experimenter to brush it. A black cloth was attached to the front edge of the box to cover the wrist of the rubber hand and the participant's arm. The rubber hand was a lifesized rubber model of a left hand. It wore a silver ring decorated with a mythological figure carved on an oval shape. Stimulation was delivered manually by the experimenter using two identical paintbrushes, with each 
stroke lasting approximately 1500 milliseconds. Participants were stimulated on the second, third and fourth finger, from the middle phalanx to the tip of the finger. The rubber hand was stimulated in the same way, either in synchrony or asynchrony with the stimulation of the participant's hand. In the synchronous condition, the participant's hand and the rubber hand were brushed at the same time and the same anatomical location. In the asynchronous condition, the hands were brushed $180^{\circ}$ out of phase in the corresponding anatomical location. Participants were instructed not to move their own hand and to observe the rubber hand for two minutes.

\section{Procedure}

Participants were asked to complete the practice phase and the first block of the perceptual matching task. After that, they repeated the task twice (block 2 and 3), while experiencing two minutes of RHI before each block. One group of participants received synchronous stimulation during the RHI while another group received asynchronous stimulation. To quantify the subjective experiences associated with the illusion, after the experiment participants completed a 27 items questionnaire (adapted from Longo et al., 2008). Participants rated different statements using a 7-point scale. Participants' naivety regarding the purpose of the experiment was assessed with a final open question. Participants were eventually debriefed and thanked for their participation.

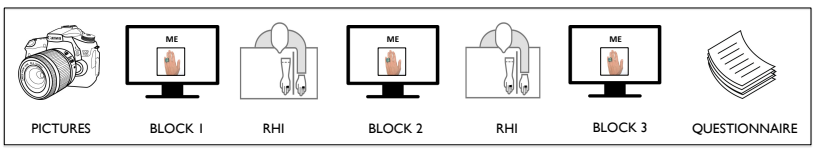

Figure 3: Timeline of the experimental procedure.

\section{Analyses}

Data were analyzed with the open-source software R (R Core Team, 2015) considering matched pair trials only. Data obtained from the practice phase were excluded.

To assess the presence of a self-prioritization effect (faster response times - RTs - and better accuracy in self-related matched trials) for both hand and self-owned ring, we adopted a bootstrapping procedure combining accuracy and RTs performance at baseline in line with Sui et al. (2012) (block 1). RTs and accuracy data for each condition and each participant were combined separately to create one data point. The data were then resampled with replacement, and the sample size was kept as the number of participants, creating a bootstrapped dataset. This procedure was repeated 2000 times, and the mean of the dataset from each instance was plotted, creating a visual representation of the estimated population mean and variation of each association condition. The self-prioritization effect was additionally tested with a signal detection approach for accuracy and with mixed-effects multiple regression models for RTs (results of these analyses are consistent with the bootstrapping procedure but are omitted for space reasons).

To evaluate the self-reported scores of ownership for the rubber hand and disownership for the real hand, we selected three subsets from the 27 items adapted from the study of Longo et al. (2008). Items from 3 to 7 reflected ownership experienced for the rubber hand (e.g., It seemed like the rubber hand belonged to me); items 14 to 18 reflected disownership experienced for one's real hand (e.g., It seemed like my hand had disappeared); items 8-9-10-27 reflected perceived location of the sensation (e.g., It seemed like I was feeling the touch of the paintbrush in the location where I saw the rubber hand being touched). Responses for each item were indicated on a seven-point Likert scale, from -3 (strong disagreement) to +3 (strong agreement). Positive responses to item 15 (It seemed like I could have moved my hand if I had wanted) were coded inversely (i.e. a response of +3 was coded as -3 ). For each participant, we computed a mean score for each category (ownership score, loss score, location score). Next, we analyzed these scores to investigate whether they were affected by the experimental condition.

RTs data across all blocks were further analyzed at the single-trial level with mixed-effects multiple regression models (Baayen, Davidson, \& Bates, 2008) using the lme4 package for R (Bates, Maechler, Bolker, \& Walker, 2014). Model comparison was performed using a stepwise approach (Bolker et al., 2009). We started from a null model containing only random terms and then we introduced fixed effects, adding main effects before interactions. The effect of factors on model fit was assessed by comparing information criteria $(\triangle \mathrm{AIC})$.

\section{Results}

An analysis of the answers to the naivety question at the end of the experiment revealed that participants did not have any understanding of the specific purpose of the study.

\section{Self-prioritization Effect}

The different distributions across the two picture-label pairs can be seen in Figure 3. For matched trials, responses to self-associations follow a distinct RT-accuracy distribution, falling in the bottom right of the figure, while response to other-associations fall towards the middle and up left of the figure. The distributions of responses for non-matching pairs overlap (not shown). Results of the bootstrap analysis show clear evidence of a self-prioritization effect. The bootstrapped dataset reveals a neat boundary between selfand other-associations. No evidence of difference between hand and ring was found. This result suggests that, before experiencing the RHI, participants' own hand and ring are included in their self-representation. 


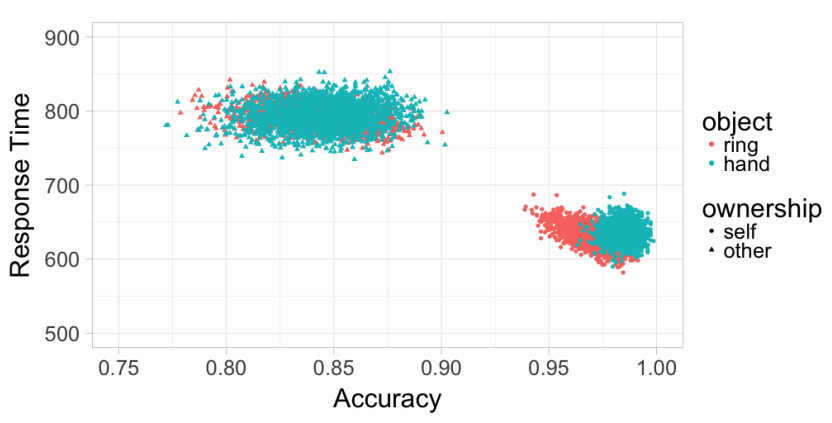

Figure 3: The self-prioritization effect in block 1 as revealed in the bootstrapped sample means for match trials. RTs are represented on the $y$-axis with accuracy on the $\mathrm{x}$ axis.

\section{Body-ownership Scores}

To establish whether participants were affected by the experimental condition (stimulation type), we next analyzed body-ownership scores. Analyses of the effect of stimulation type on explicit judgments showed a significant difference in body-ownership scores between synchronous and asynchronous participants, $\mathrm{F}(1,39)=14.98, p=.0004$, $\eta^{2}=.27$, with the synchronous condition yielding higher ownership scores, $\mathrm{M}=0.98(\mathrm{SD}=1.59)$, than the asynchronous condition, $\mathrm{M}=-0.96(\mathrm{SD}=1.62)$. Similarly, we found a significant difference in location scores, F $(1,39)$ $=30.43, p<.0001, \eta^{2}=.43$, with the synchronous group resulting in higher location scores, $\mathrm{M}=1.57(\mathrm{SD}=1.07)$ than the asynchronous one, $\mathrm{M}=-0.55(\mathrm{SD}=1.38)$. In contrast, loss scores were not affected by condition $\mathrm{F}(1,39)$ $=0.54, p=0.46, \eta^{2}=.01$. Mean score and SD for the synchronous and asynchronous participants were respectively $\mathrm{M}=-0.30(\mathrm{SD}=1.50)$ and $\mathrm{M}=-0.65(\mathrm{SD}=$ $1.38)$.

\section{Response Times}

First, baseline RTs (block1) for matched trials were analyzed to exclude a possible initial difference between the group of participants receiving synchronous stimulation and the group receiving asynchronous stimulation. Results from a mixed model including participants and objects as random factors and stimulation type (synchronous vs asynchronous) as a fixed factor did not show a significant effect of the experimental condition, $\mathrm{b}=-30.04, \mathrm{t}=-1.005, \mathrm{p}=0.32$.

Next, to assess the effect of stimulation type on participants' performance across the three blocks of the task, a mixed-effects model was fitted to the RTs data. The random effects structure included participants and objects, while stimulation type, trial number and the interaction between them were treated as fixed factors. A comparison between a first model including random effects only (Resid. Df: 4669, Resid Dev: 62034, $\Delta$ AIC: 316, AIC weight:0), a second model considering main effects of condition and trial number (Resid. Df: 4667, Resid Dev: 61725, $\triangle$ AIC: 11.1, AIC weight:0) and a third model considering the interaction between them (Resid. Df: 4666, Resid Dev: 61712, $\triangle$ AIC:
0, AIC weight:1) revealed that the model with the interactive term was the best.

Results from the interactive model showed that the experimental condition (type of stimulation) affected the learning rates of participants. Response times decreased across trial number, $\mathrm{b}=-.63, \mathrm{t}=-17.859, \mathrm{p}<0.001$, but decreased less in the synchronous condition than in the asynchronous condition, $\mathrm{b}=.25, \mathrm{t}=3.61, \mathrm{p}=.0003$ (interaction between trial number and condition). In other words, participants experiencing the synchronous stimulation were slower in learning across trials compared to the asynchronous group (see Figure 4).

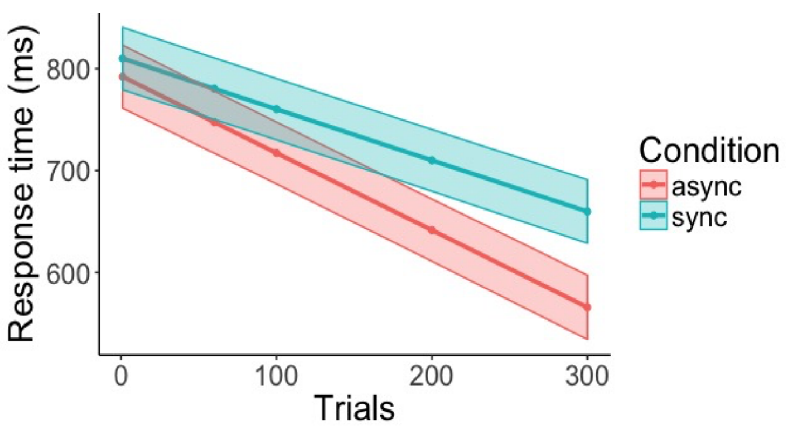

Figure 4: Participants' learning rates across (all blocks).

We then run a second analysis on RTs after the RHI (blocks 2 and 3) considering also the possible effects of ownership category (whether the picture-label pair belonged to the self-ownership or the other-ownership category) and object type (whether the object presented as stimulus was the hand, the hand with the ring or the ring alone).

A comparison between a first model including random effects only (Resid. Df: 3151, Resid Dev: 41682, $\triangle$ AIC: 54.4, AIC weight: 0), a second model considering the main effect of stimulation type (sync vs async) (Resid. Df: 3150, Resid Dev: 41679, $\triangle$ AIC: 53.5, AIC weight: 0) a third model adding ownership category (Resid. Df: 3149 , Resid Dev: 41628, $\triangle$ AIC: 3.7 , AIC weight: 0.08 ), a fourth model adding object type (Resid. Df:3147, Resid Dev: 41621, $\triangle$ AIC: 0.9 , AIC weight: 0.33), an interactive model considering the interaction between stimulation type and ownership (Resid. Df: 3146, Resid Dev: 41618, $\triangle$ AIC: 0, AIC weight: 0.53 ) and a final model considering the interaction between stimulation type and object type (Resid. Df: 3145, Resid Dev: 41620, $\Delta$ AIC: 4.5, AIC weight: 0.05) revealed that the model with the interactive term stimulation type * ownership was the best.

Results from the final model (see Table 1) showed a main effect of ownership $(b=-177.87, t=-16.568, p<0.001)$ and a main effect of condition $(b=61.56, t=2.42, p=0.02)$. In other words, self-related pairings were processed faster than other-related ones (=self prioritization effect) but response times were slower in the synchronous than in the asynchronous condition for both self- and other-ownership matched trials (see Figure 5). We found no evidence that the effect was modulated by object type. 
Table 1: Results of the best fitting mixed-effect model.

\begin{tabular}{|l|l|l|l|l|l|}
\hline & $\mathrm{b}$ & $\mathrm{SE}$ & $\mathrm{df}$ & $\mathrm{t}$ value & $\mathrm{p}$ value \\
\hline (Intercept) & 690.507 & 13.613 & 53.709 & 50.723 & $<2 \mathrm{e}-16^{* * *}$ \\
\hline $\begin{array}{l}\text { Ownership (centred; Self }= \\
0.5, \text { Other =-0,5) }\end{array}$ & -177.876 & 10.736 & 40.615 & -16.568 & $<2 \mathrm{e}-16^{* * *}$ \\
\hline $\begin{array}{l}\text { Condition (centred; Sync= } \\
0.5, \text { Async = -0,5) }\end{array}$ & 61.564 & 25.437 & 40.930 & 2.420 & $0.0200 *$ \\
\hline Condition * Ownership & -37.344 & 21.472 & 40.614 & -1.739 & 0.0896. \\
\hline Object type: Ring & 13.280 & 7.418 & 3078.204 & 1.790 & 0.0735. \\
\hline Object type: Hand with ring & -5.480 & 8.098 & 3077.192 & -0.677 & 0.4987 \\
\hline
\end{tabular}

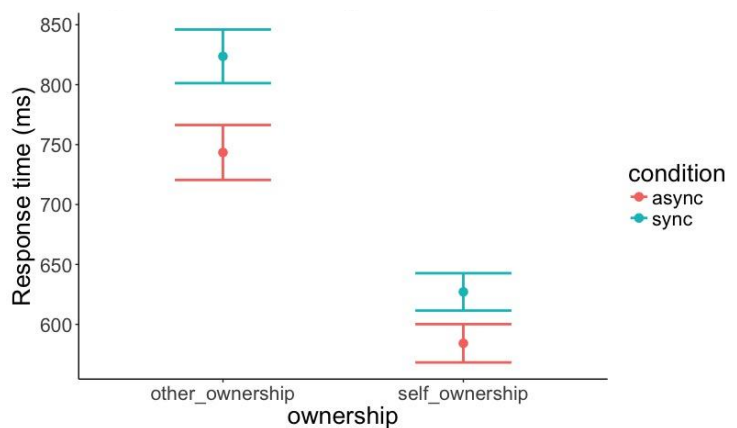

Figure 5: Response times on self- and other-ownership matched trials are slower in the Synchronous than in the Asynchronous condition (blocks 2-3).

These results provide support to both the Ownership hypothesis (slower RTs in the synchronous group when perceiving objects belonging to someone else) and the Disownership hypothesis (slower RTs in the synchronous group when perceiving self-owned objects). Overall, they indicate that the experience of object ownership can indeed be based on the incorporation of objects in our body image.

\section{Conclusions}

Results of our experimental study show that the sense of object ownership, as measured through the selfprioritization effect, was modulated by the rubber hand illusion and, in particular, was attenuated in the group of participants who received the synchronous stimulation relative to those who received the asynchronous one. Moreover, we have shown that changing the "bodily" representation of the self can affect its "conceptual" representation, since undermining the bodily self (via body manipulation) has cascade effects on conceptual representations, at least when an object, like a ring, has been systematically associated with a body-part.

Reviewing the state of art at the time on the neural basis of body ownership, Botvinick observed that: "the feeling of ownership that we have for our bodies clearly does not extend to, for example, the fork we use at dinner." (Botvnick, 2004: 783). Although we clearly do not mistake our fork for our own hand when eating at the restaurant, results of this study raise the intriguing possibility that its inclusion into one's own body schema might potentially ground a sense that is our (psychological owned) fork.

Whether, however, the me is mine view generalizes beyond items like ornaments, clothes or shoes that are systematically associated with our body is left for future research.

\section{References}

Abed, R. T., \& Fewtrell, W. D. (1990). Delusional misidentification of familiar inanimate objects a rare variant of Capgras Syndrome. The British Journal of Psychiatry, 157(6), 915-917.

Aglioti, S., Smania, N., Manfredi, M., \& Berlucchi, G. (1996). Disownership of left hand and objects related to it in a patient with right brain damage. Neuroreport, 8(1), 293-296.

Alves, P. N., Reimão, S., Campos, C., Maruta, C., Rocha, L., Sá, G., ... \& Martins, I. P. (2016). Loss of object ownership feeling following a left hemisphere infarct. Cortex, 84, 132-134.

Armel, K. C., \& Ramachandran, V. S. (2003). Projecting sensations to external objects: evidence from skin conductance response. Proceedings of the Royal Society of London. Series B: Biological Sciences, 270(1523), 1499-1506.

Baayen, R. H., Davidson, D. J., \& Bates, D. M. (2008). Mixed-effects modeling with crossed random effects for subjects and items. Journal of memory and language, 59(4), 390-412.

Bates, D., Mächler, M., Bolker, B., \& Walker, S. (2014). Fitting linear mixed-effects models using lme4. arXiv preprint arXiv: 1406.5823.

Beggan, J. K. (1992). On the social nature of nonsocial perception: The mere ownership effect. Journal of personality and social psychology, 62(2), 229-237.

Belk, R. W. (1988). Possessions and the extended self. Journal of consumer research, 15(2), 139-168.

Blanke, O., Slater, M., \& Serino, A. (2015). Behavioral, neural, and computational principles of bodily selfconsciousness. Neuron, 88(1), 145-166.

Bolker, B. M., Brooks, M. E., Clark, C. J., Geange, S. W., Poulsen, J. R., Stevens, M. H. H., \& White, J. S. S. (2009). Generalized linear mixed models: a practical guide for ecology and evolution. Trends in ecology \& evolution, 24(3), 127-135.

Botvinick, M. (2004). Probing the neural basis of body ownership. Science, 305(5685), 782-783.

Botvinick, M., \& Cohen, J. (1998). Rubber hands 'feel' touch that eyes see. Nature, 391(6669), 756-756.

Cunningham, S. J., Turk, D. J., Macdonald, L. M., \& Macrae, C. N. (2008). Yours or mine? Ownership and memory. Consciousness and cognition, 17(1), 312-318.

Ehrsson, H.H. (2012). The concept of body ownership and its relation to multisensory integration. In: The New Handbook of Multisensory Processes, B.E. Stein (Ed.), MA: MIT Press (Cambridge). 
della Gatta, F., Garbarini, F., Puglisi, G., Leonetti, A., Berti, A., \& Borroni, P. (2016). Decreased motor cortex excitability mirrors own hand disembodiment during the rubber hand illusion. Elife, 5, e14972.

Humphreys, G. W., \& Sui, J. (2015). The salient self: Social saliency effects based on self-bias. Journal of Cognitive Psychology, 27(2), 129-140.

James, W. (1890). Principles of psychology. New York: Macmillan.

Kim, K., \& Johnson, M. K. (2014). Extended self: spontaneous activation of medial prefrontal cortex by objects that are 'mine'. Social cognitive and affective neuroscience, 9(7), 1006-1012.

Lane, T., Yeh, S. L., Tseng, P., \& Chang, A. Y. (2017). Timing disownership experiences in the rubber hand illusion. Cognitive research: principles and implications, 2(1), 4 .

Longo, M. R., Schüür, F., Kammers, M. P., Tsakiris, M., \& Haggard, P. (2008). What is embodiment? A psychometric approach. Cognition, 107(3), 978-998.

Ma, K., \& Hommel, B. (2015). Body-ownership for actively operated non-corporeal objects. Consciousness and cognition, 36, 75-86.

Maister, L., Slater, M., Sanchez-Vives, M. V., \& Tsakiris, M. (2015). Changing bodies changes minds: owning another body affects social cognition. Trends in cognitive sciences, 19(1), 6-12.

Merrill, T. W. (1984). Property rules liability rules and adverse possession. Nw. UL Rev., 79, 1122.

Morewedge, C. K., \& Giblin, C. E. (2015). Explanations of the endowment effect: an integrative review. Trends in cognitive sciences, 19(6), 339-348.

Peck, J., \& Shu, S. B. (Eds.). (2018). Psychological ownership and consumer behavior. Springer.

Pierce, J. L., Kostova, T., \& Dirks, K. T. (2003). The state of psychological ownership: Integrating and extending a century of research. Review of general psychology, 7(1), 84-107.

Scorolli, C., Borghi, A.M., \& Tummolini, L. (2018). Cues of control modulate the ascription of object ownership. Psychological research, 82(5), 929-954.

Sui, J., He, X., \& Humphreys, G. W. (2012). Perceptual effects of social salience: evidence from self-prioritization effects on perceptual matching. Journal of Experimental Psychology: Human perception and performance, 38(5), 1105-1117.

Turk, D. J., van Bussel, K., Brebner, J. L., Toma, A. S., Krigolson, O., \& Handy, T. C. (2011a). When "it" becomes "mine": Attentional biases triggered by object ownership. Journal of cognitive neuroscience, 23, 37253733

Turk, D. J., Van Bussel, K., Waiter, G. D., \& Macrae, C. N. (2011b). Mine and me: Exploring the neural basis of object ownership. Journal of cognitive neuroscience, 23(11), 3657-3668.

Ye, Y., \& Gawronski, B. (2016). When possessions become part of the self: Ownership and implicit self-object linking. Journal of Experimental Social Psychology, 64, 72-87.

Weser, V., Finotti, G., Costantini, M., \& Proffitt, D. R. (2017). Multisensory integration induces body ownership of a handtool, but not any handtool. Consciousness and cognition, 56, 150-164. 